\title{
THE DIAMETER-REDSHIFT RELATION
}

\author{
W. A. BAUM \\ Lowell Observatory, Flagstaff, Ariz., U.S.A.
}

\begin{abstract}
An observational investigation of the angular diameter-redshift relation for galaxies is described, using results from a 'photographic galaxy synthesizer'. The observations give a value $q_{0}=+0.3$ for the deceleration parameter.
\end{abstract}

Among the possible tests of world models, there is the relationship between the apparent angular diameters of distant galaxies and their redshifts. This relationship has not been used, because we have not previously had a practical way to measure the true diameters of distant galaxies without incurring serious errors from optical and atmospheric effects. This paper introduces a new method and presents a newly derived value for the deceleration parameter $q_{0}$.

In order to reach a distance at which the difference from one world model to another will be substantially larger than the scatter of data arising from intrinsic differences among the galaxies sampled, we must deal with clusters of galaxies out to the most distant ones known. At such a distance, the galaxies are only subtly distinguishable from faint stars in photographs taken with large Earth-based telescopes. Because of this, it has been suggested that the angular sizes of clusters of galaxies should be used instead of the apparent angular diameters of the individual galaxies themselves. Such a procedure has to be ruled out, however, because we cannot assume a cluster of galaxies to be unchanging in spatial distribution. We have to use the apparent angular diameters of individual galaxies themselves, if this test of world models is to be valid. Present knowledge of the stellar content of elliptical galaxies is sufficient to justify the belief that evolutionary changes in luminosity distribution will be too small to alter significantly the results of a cosmological test based on diameters.

A simple device was made to attack this problem. It was used in place of a conventional plate holder at the prime focus of the Hale telescope at Palomar. A photographic plate is cut into two halves that are put into two adjacent plate holders. A cluster of galaxies is focused and centered on one of the halves, which remains stationary in the focal plane. The adjacent star field is imaged on the other half, which is moved continuously in-and-out of focus by a cam-driven mechanism. Stars are thereby built up into fuzzy images resembling E0 galaxies. The device is therefore a photographic galaxy synthesizer. The synthetic galaxy images can later be compared with the real galaxy images being photographed simultaneously on the stationary half of the plate.

By the use of this technique, optical and atmospheric effects cancel out. Imagine for a moment an ideal star image - one that is undisturbed by optics and atmosphere. It would then be an extremely small point through which a conical bundle of light rays pass. Owing to the prime focus cage of the Hale telescope, the cross-section of 
this cone would be an annulus with a dark center. If a photographic plate is moved along the optic axis from a plane behind the focus to a plane ahead of the focus, the integrated exposure on an ideal star would be a diffuse disk with a central concentration. If the plate is driven cyclically through focus in this manner at a suitably chosen non-linear rate, the intensity profile of the resulting synthetic galaxy can be made similar to the profiles of real E0 galaxies. Although the luminosity distributions of E0 galaxies are not quite identical, it is fair to say that the profile produced by the galaxy synthesizer does not differ from the profiles of real galaxies more than they differ among themselves. If the moving plate oscillates through focus a large number of times during the total exposure, the build up of the synthetic galaxy image is not modified by any unwanted photographic intermittency effect. In practice, of course, the real star images are not ideal points; they are convolved with the same optical and atmospheric effects as the real galaxy images which are being photographed simultaneously.

It is important to point out that the galaxy synthesizer method provides a measurement of the relative metric diameters of galaxies, as distinct from their isophotal diameters. Otherwise it would not be so satisfactory a test of world models, because it would depend heavily on corrections for observational effects. The derived diameters are metric, because the effective width of the synthetic profile function (analogous to the half-power width of a Gaussian function) is a mechanically adjustable linear dimension in the focal plane and is independent of the brightness of the star that produces the synthetic image. The aim is to determine when the mechanical amplitude of the generating function of the galaxy synthesizer produces a correct match to a real galaxy whose diameter we want to know. The observing technique is to obtain

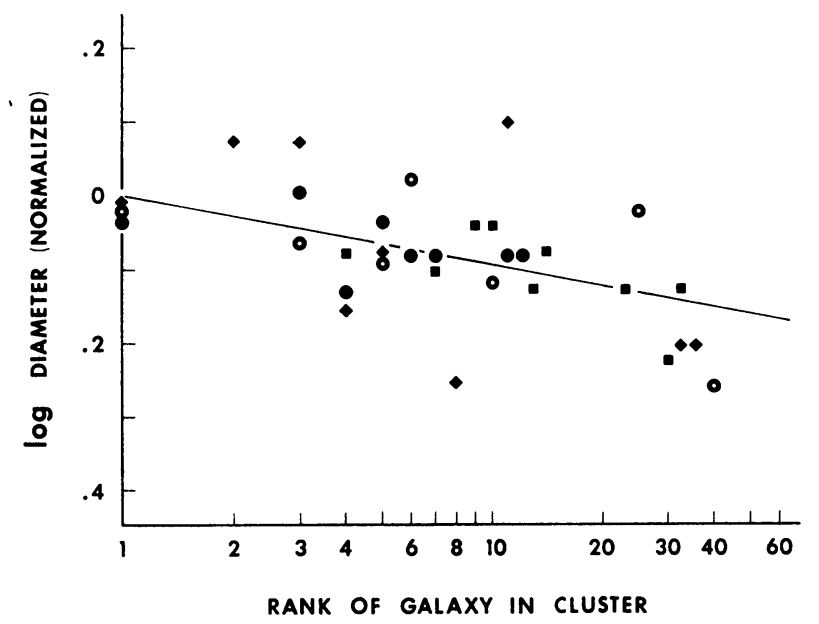

Fig. 1. Dependence of metric diameter on rank in each of the four clusters investigated. Squares: CL $2322+1425, z=0.044$; Open circles: CL $1520+2754, z=0.072$; Diamonds: CL $0024+1654$, $z=0.29$; Closed circles: CL $1410+5224, z=0.46$. For each cluster the ordinate has been normalized to unit diameter at rank $=1$. The vertical scatter of $0.065(\mathrm{rms})$ in $\log d$ is due partly to intrinsic differences among the galaxies and partly to errors of measurement. 
six or seven pairs of plates using stepped increments (of about the square root of 2) in the amplitude of the synthetic generating function. The synthetic images on the moving plate of a particular pair are then compared with the real images of the non-moving plate of the same pair in order to determine for individual real galaxies whether the synthetic ones are too small or too large. We must limit attention to images of the same apparent brightness if the match or mismatch is to be judged accurately. In most cases, the adjacent star fields provide an adequate range of brightnesses among the synthetic images to provide a good choice. The most effective instrument for judging the match of images is an ordinary spectrum comparator, which enables the two images, real and synthetic, to be placed side by side in the field of a single eyepiece.

Thus far, the apparent angular diameters of galaxies in four clusters have been measured by this method in the photovisual region. These four have redshifts ranging from 0.04 to 0.46 , thereby spanning a factor of 10 . An average of eight galaxies per cluster were measured, ranging in rank from the first brightest to the 40th brightest. Figure 1 shows the correlation between metric diameter and rank. The data for the four clusters are combined here, all clusters having been normalized to unit diameter, referred to the first brightest. All four clusters look comparably populous, and this diagram shows no systematic difference in the dependence on rank. The Scott selection effect will evidently be less of a hazard than it is for magnitude-redshift data.

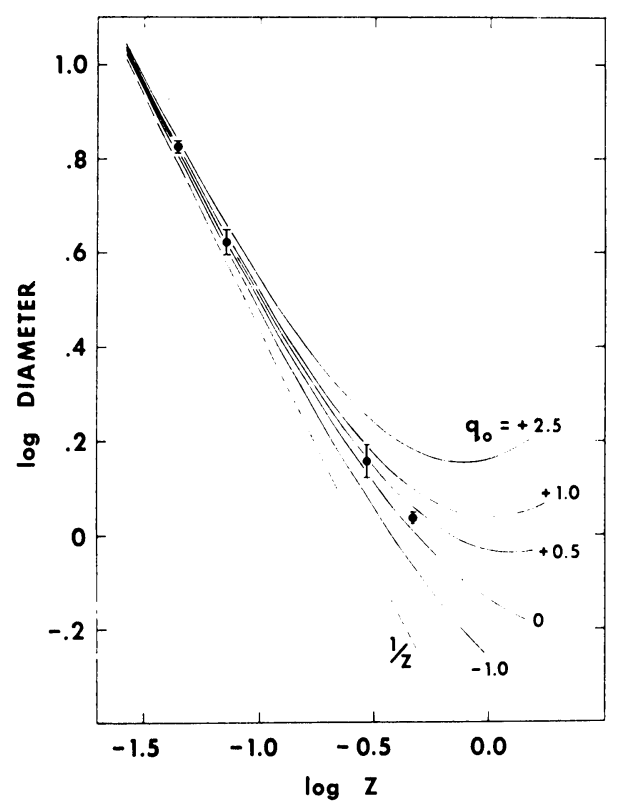

Fig. 2. Comparison of diameter-redshift data with world models. Each cluster is represented here by a single point based on the data in Figure 1 before normalization. Error bars represent standard errors based on the scatter of the points for the individual clusters in Figure 1. The curve for $q_{0}=-1.0$ represents the steady-state model, the range between $q_{0}=0$ and $q_{0}=+0.5$ represents the open-universe case of the exploding model, while values of $q_{0}$ greater than +0.5 represent the closed-universe case of the exploding model. 
When the data for each cluster are reduced to a single characteristic diameter, we obtain the correlation with redshift plotted in Figure 2. The four points are overlaid here with lines representing possible world models. Error bars associated with the points represent standard errors based on the rms scatter of diameters and the number of galaxies measured in each cluster.

The curves for the various world models are identified by values of the deceleration parameter $q_{0}$ and are adapted from a paper by Sandage (1961). The curve for $q_{0}=-1.0$ represents the steady-state model, while positive values of $q_{0}$ represent exploding models. The observed points fit a value of $q_{0}=+0.3$, which is in the range of open universes that will not later collapse. This result is at variance with existing redshiftmagnitude data, including my own that reach to $z=0.46$ and that imply $q_{0} \geqq+1.0$. Since magnitude data used in plotting a redshift-magnitude relation depend somewhat upon the effective diameters of magnitude measurement, the results presented here could be used to derive a correction to existing redshift-magnitude results. Such a correction, however, would not be large and would tend slightly to increase the disparity rather than reduce it. I should also point out that the disparity is not in the direction that one might explain by a possible non-transparency of space.

My present feeling about these diameter-redshift results is that they are fairly reliable and that the disparity with the redshift-magnitude data may not disappear easily. It is going to be very important to observe additional clusters and perhaps to re-observe one of the more distant clusters of Figure 1 in more nearly the same nonredshifted wavelength region that the nearer clusters have been observed in. Meanwhile, $q_{0}=+0.3$ is what the diameter-redshift data evidently imply.

\section{Reference}

Sandage, A. R.: 1961, Astrophys. J. 133, 355.

\section{Discussion}

Miley: What would you estimate as the limits to your value of $q_{0}$ as determined from this method? Baum: Perhaps \pm 0.2 , but it is too early to be definite. 